Agron. Mesoam. 25(2):297-311. 2014

ISSN:2215-3608 doi 10.15517/am.v25i2.15435

\title{
EVALUACIÓN DEL ENSILAJE DEL PASTO RATANA (Ischaemum indicum HOUTT.) CON TRES DIFERENTES ADITIVOS ${ }^{1}$
}

\author{
Carlos Boschini-Figueroa ${ }^{2}$,Luis Pineda-Cordero², Pablo Chacón-Hernández ${ }^{2}$
}

\begin{abstract}
RESUMEN
Evaluación del ensilaje del pasto Ratana (Ischaemum indicum Houtt.) con tres diferentes aditivos. El objetivo de este trabajo fue evaluar las características del proceso de fermentación del pasto Ratana (Ischaemum indicum Houtt.) y la calidad del ensilado, al agregar tres aditivos. El experimento se llevó a cabo en la provincia de Limón, Costa Rica, durante el periodo de julio a diciembre del 2011; zona con una temperatura de $24,5^{\circ} \mathrm{C}$; una altura de $249 \mathrm{msnm}$ y precipitación de 4577,2 $\mathrm{mm}$ anuales distribuidos principalmente de mayo a diciembre. Se empleó un diseño de bloques completos al azar, con cuatro repeticiones y nueve tratamientos: ensilado con melaza, maíz molido o pulpa de cítricos deshidratada como aditivos, en tres niveles de adición ortogonales. Las pérdidas de material más importantes se produjeron con melaza o pulpa de cítricos $(\mathrm{p}<0,05)$ con disminuciones en base fresca de $13,27 \%$ y $14,42 \%$, respectivamente; mientras que el ensilaje con maíz molido tuvo mermas de $11,68 \%$. No se encontró ninguna diferencia $(\mathrm{p}>0,05)$ en los porcentajes de materia seca (MS), fibra neutrodetergente (FND), fibra ácido detergente (FAD), hemicelulosa y cenizas en los ensilajes de ninguno de los tratamientos. La proteína cruda (PC), celulosa y extracto etéreo $(\mathrm{EE})$ difirieron según el porcentaje de aditivo agregado $(\mathrm{p}<0,05)$. Los niveles de $\mathrm{pH}$ encontrados fueron estadísticamente menores $(\mathrm{p}<0,05)$ en los tratamientos que incluyeron melaza $(4,84)$, que en los ensilados con maíz $(5,10)$ o citropulpa $(5,02)$. Finalmente, todos los tratamientos analizados presentaron características organolépticas correspondientes a ensilados de buena calidad.
\end{abstract}

Palabras clave: melaza en ensilaje, maíz en ensilaje, citropulpa en ensilaje.

\begin{abstract}
Evaluation of Ratana grass (Ischaemum indicum Houtt.) silage with three different kinds additives. The objective of the present work, was to evaluate the characteristics of the fermentation process of Ratana Grass (Ischaemum indicum Houtt.) and the quality of the ensilage produced, when three different additives were added. The experiment was carried out at Limón, Costa Rica, between the months of July and December, at an area located 249 m.a.s.1., with a mean temperature of $24.5^{\circ} \mathrm{C}$ and $4577.2 \mathrm{~mm}$ of rain distributed mainly between May and December. A randomized complete block design, with four repetitions and nine treatments was used with the following additives: soilage with molasses, ground corn or dehydrated citrus pulp on three orthogonal levels of addition. Molasses and dehydrated citrus pulp produced the most important losses $(\mathrm{p}<0.05)$ with fresh matter reductions of $13.27 \%$ and $14.42 \%$ respectively; while the treatments with ground corn reported $11.68 \%$ of fresh matter losses. No differences were obtained $(p>0.05)$ for dry matter (DM), neutral detergent fiber (NDF), acid detergent fiber (ADF), hemicellulose and ashes percentages on any treatment. Crude protein $(\mathrm{CP})$, cellulose and ether extract (EE) differed according to the amount of additive used $(\mathrm{p}<0.05)$. $\mathrm{pH}$ levels found were statistically lower for the molasses treatments (4.84), compared to the ground corn (5.10) or dehydrated citrus pulp (5.02) treatments. Finally, all analyzed treatments showed organoleptic characteristics corresponding to good quality silages.
\end{abstract}

Keywords: silage with molasses, ground corn in silage, citrus pulp in silage.

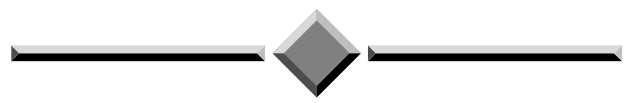

Recibido: 22 de agosto, 2013. Aceptado: 30 de junio, 2014. Proyecto de investigación VI-737-B1-074 "Evaluación de forrajes tropicales y desarrollo de cultivos forrajeros. Universidad de Costa Rica, San José, Costa Rica.

2 Estación Experimental "Alfredo Volio Mata”, Facultad de Ciencias Agroalimentarias, Universidad de Costa Rica. San José, Costa Rica. carlos.boschini@ucr.ac.cr, luis.pineda@ucr.ac.cr, pablo.chacon@ucr.ac.cr 


\section{INTRODUCCIÓN}

En los sistemas de producción animal resulta imprescindible cubrir los requerimientos nutricionales de los animales durante todo el año y disponer de reservas alimenticias capaces de garantizar una estabilidad productiva. Estas reservas de alimento deben considerarse no solo en términos de cantidad sino también de calidad, donde predomine el interés de eliminar el déficit de nutrientes a partir del forraje generado en los sistemas de producción utilizados (Ojeda et al., 2006).

Los forrajes constituyen la fuente más económica de nutrientes para el ganado, existiendo diversas especies forrajeras adaptadas a las diferentes zonas agroecológicas, pero con deficiencia en su uso y aprovechamiento lo que implica frecuentes improvisaciones para solucionar dificultades debido a la escasez (Lara, 2011).

Se estima que para el año 1988, en Costa Rica, el pasto Ratana, Retana o Rotana (Ischaemum indicum Houtt, mencionado también por la literatura como Ischaemum ciliare Retz. o Pheleum indicum) ocupaba un $41,5 \%$ de las áreas de pasto cultivadas de la Región Huetar Norte y el 43,1\% en la Región Huetar Atlántica, siendo actualmente aún muy utilizado en sistemas de producción de bajos insumos y ganaderías extensivas (Villarreal, 1992). Aunque tiene una propagación masiva, esta especie se caracteriza por ser más conocida por la experiencia empírica que por el trabajo técnicocientífico (Villarreal, 1992; Villareal, 2010).

Aunque para este forraje se mencionan características indeseables, como baja productividad, sistema radicular superficial, así como floración profusa y prolongada, durante la época seca, la especie I. indicum Houtt. puede constituir una opción forrajera en ganaderías extensivas donde por razones técnicas, económicas o preferencias del productor, la introducción de otras especies no ha sido factible o exitosa (Arosemena et al., 1996; Villareal, 2010).

Una pastura de Ratana puede producir en la zona de San Carlos, Costa Rica, bajo sistemas de cosecha cada 35 días un promedio de $462 \mathrm{~kg}$ de materia seca/ ha/corte y $283 \mathrm{~kg}$ de materia seca/ha/corte en la época de mínima y máxima precipitación, respectivamente Villarreal (1992), material que según las condiciones podría conservarse para la suplementación del ganado. Siendo este el caso, el uso del ensilaje permitiría al productor intensificar la productividad de la tierra y de los animales de forma independiente (Wattiaux, s.f.).

Por medio del ensilaje se puede almacenar y reservar el alimento para su uso posterior con pérdidas mínimas de calidad nutricional (Garcés et al., 2004; Qamar, 2009; Reyes et al., 2009; Lara, 2011; Wattiaux, s.f.). En el caso de las zonas tropicales y subtropicales, el ensilado de cultivos forrajeros o subproductos industriales podría ser una contribución importante para optimizar el funcionamiento de los sistemas de producción animal (Garcés et al., 2004). Sin embargo, debe tomarse en cuenta la composición química del material a ensilar, ya que esta juega un papel importante en la determinación del potencial del material a ser ensilado, conservándose de mejor manera forrajes con altos niveles de azúcares fermentables, bajo nivel de proteína, baja capacidad de amortiguación del $\mathrm{pH}$ y un mínimo contenido de materia seca (MS) (Nisa et al., 2008). Estas condiciones difícilmente se presentan con los materiales disponibles en las zonas tropicales (Bergamaschine et al., 2006).

Como estrategia para favorecer el proceso de conservación en los ensilajes, se ha venido implementando el uso de diversos componentes conocidos como aditivos que tienen la función de suplir aquellos faltantes en el material utilizado y reducir las pérdidas de forraje (Bolsen et al., 1996; Santos et al., 2010). Los aditivos son productos que estabilizan el ensilado, ya sea por acidificación, limitando el crecimiento de microorganismos, o bien estimulando la fermentación láctica (Lara, 2011). Dentro de estos últimos se pueden emplear fuentes de azúcares solubles que las bacterias utilizan para producir ácido láctico como el maíz triturado, melaza (Pereira et al., 1999; Qamar, 2009) o pulpa de cítricos (Pereira et al., 1999, Garcés et al., 2004); contribuyendo de esta forma a la creación de condiciones óptimas que permitan la conservación del ensilaje (Lara, 2011).

El objetivo de este trabajo fue evaluar las características del proceso de fermentación del pasto Ratana (Ischaemum indicum Houtt.) y la calidad del ensilado, al agregar tres aditivos. 


\section{MATERIALES Y MÉTODOS}

\section{Ubicación y manejo del forraje}

El experimento se llevó a cabo entre los meses de julio a diciembre, en la Finca La Curia, localizada en el distrito de Cariari, cantón de Pococí, provincia de Limón, la cual tiene una altitud de $249 \mathrm{msnm}$, con una precipitación media anual de 4577,2 mm, distribuida en los meses de mayo a diciembre del 2011. La temperatura promedio es de $24,5^{\circ} \mathrm{C}$ (máxima de $28,7{ }^{\circ} \mathrm{C}$, mínima de $20,2{ }^{\circ} \mathrm{C}$ ), una humedad relativa media del $87 \%$ y una evaporación de $3,6 \mathrm{~mm}$ (IMN, comunicación personal, 2013). Ningún tipo de fertilización o enmienda fue aplicado al suelo antes o durante el desarrollo del experimento. El forraje de $I$. indicum se cosechó a una edad de rebrote de setenta días por medio de una motoguadaña para luego ser recolectado y proceder con el llenado de las bolsas.

\section{Preparación de las bolsas}

Para la conservación del forraje, se utilizaron bolsas de polietileno de siete milésimas de pulgada de grosor (medida disponible comercialmente), de color negro, con un tamaño de $91 \mathrm{~cm}$ de ancho por 125 $\mathrm{cm}$ de alto, con una capacidad teórica para $60 \mathrm{~kg}$ de forraje verde según las especificaciones brindadas por el fabricante. Además, cada bolsa fue acondicionada en el extremo inferior con un drenaje para los posibles efluentes producidos, dicho desagüe consistió en un trozo de manguera plástica de $1 \mathrm{~cm}$ de diámetro interno y $30 \mathrm{~cm}$ de longitud. El acondicionamiento de la salida de los efluentes se llevó a cabo perforando el extremo inferior de la bolsa para introducir 10 $\mathrm{cm}$ de manguera, ahusando luego la sección final de plástico alrededor de ella, para en seguida atar el trozo de manguera en el interior de la bolsa con 1,5 m de manila de nylon. Posteriormente, se recubrió con cinta adhesiva de tela el extremo de la bolsa y parte de la manguera con el fin de fijar el desagüe fuertemente a la bolsa, dejando un extremo de $20 \mathrm{~cm}$ para doblar y atar a $10 \mathrm{~cm}$ la parte externa de la manguera y contener la salida de los líquidos de la bolsa que se desprendieran durante el proceso de fermentación, de forma tal que fuera posible extraer y medir la cantidad de efluentes producidos durante el periodo experimental.

\section{Proceso de llenado de las bolsas}

El forraje fue depositado dentro de las bolsas inmediatamente después de ser segado sin realizársele ningún tratamiento previo al proceso de conservación; el material cortado se fue colocando y compactando inmediatamente de forma manual en las bolsas en capas de 20-25 cm de alto; repitiendo este proceso de forma sucesiva hasta que únicamente quedara bolsa desocupada para poder recoger los bordes. Posterior al proceso de llenado y compactado, a cada bolsa se le introdujo una manguera conectada a una bomba de vacío calibrada a $-0,052 \mathrm{MPk}$ (equivalente a -38 cm $\mathrm{Hg},-15$ psi o una atmósfera de vacío) para una compactación final del material (cerca de un 40\%) y extracción del aire residual dentro de la bolsa. Previo al cierre de la bolsa se agregaron los aditivos en las proporciones previstas por cada tratamiento experimental (cuyas proporciones se detallan más adelante), de acuerdo al peso del forraje ingresado y su contenido de materia seca valorada con anterioridad en el laboratorio. Finalmente, el extremo de la bolsa se amarró con cuerda de $3,2 \mathrm{~mm}$ de grosor mediante un doble nudo, doblando a continuación el extremo libre de la bolsa hacia abajo para formar un moño con la misma que impidiera un eventual ingreso de agua, polvo o insectos al interior de la bolsa.

El contenido de forraje en cada bolsa fue pesado, los tratamientos fueron rotulados en las bolsas y ellas se colocaron en una tarima elevada a $20 \mathrm{~cm}$ sobre el nivel del piso, de manera que permitiera la remoción de los efluentes producidos durante el proceso de fermentación del material.

\section{Determinación del nivel de aditivo de cada tratamiento}

Los aditivos empleados fueron melaza, maíz molido y Citrocom (alimento comercial peletizado a base de pulpa de cítricos deshidratada), adicionados al forraje en tres proporciones diferentes, identificadas como nivel 1,2 y 3 .

Para fijar la adición del porcentaje más bajo de cada agregado se siguieron dos vías diferentes; para la melaza se decidió agregarla hasta un nivel que permitiera obtener un mínimo de azúcares en la mezcla a ensilar de $10 \%$; dado que el contenido de carbohidratos 
no fibrosos en el pasto fue de $17,09 \%$ de la materia seca (MS), el nivel mínimo de adición fue de $0 \%$. Por su parte, el Citrocom y el maíz molido fueron agregados al forraje de forma que se alcanzara un mínimo de $20 \%$ de materia seca en el material a ser conservado; al haber sido determinado un $\%$ de MS en el pasto de $27,16 \%$, los niveles más bajos de estos aditivos fueron igualmente de $0 \%$. A partir de estos niveles base de adición, se decidió fijar los tres agregados a evaluar en niveles ortogonales correspondientes a $0 \%, 2 \%$ y $4 \%$ cada uno, correspondientes a los de inclusión 1,2 y 3 , respectivamente.

\section{Diseño experimental y toma de muestras}

Se utilizó un diseño de bloques completos al azar (Steel y Torrie, 1988) con cuatro repeticiones y nueve tratamientos en arreglo factorial. Los factores fueron tres diferentes aditivos: melaza, maíz molido y Citrocom peletizado, cada uno en tres niveles de inclusión ortogonales (niveles de inclusión 1, 2 y 3). Con base en los análisis bromatológicos iniciales, realizados una semana antes de la elaboración de los ensilajes, se determinó el contenido de materia seca (MS), proteína cruda (PC), extracto etéreo (EE), cenizas (CEN), fibra neutro detergente (FND), fibra ácido detergente (FAD), lignina, hemicelulosa y celulosa, la proteína cruda incrustada en la fibra neutro detergente (PCIFND) y los carbohidratos no fibrosos (CNF) mediante la siguiente fórmula (NRC, 2001):

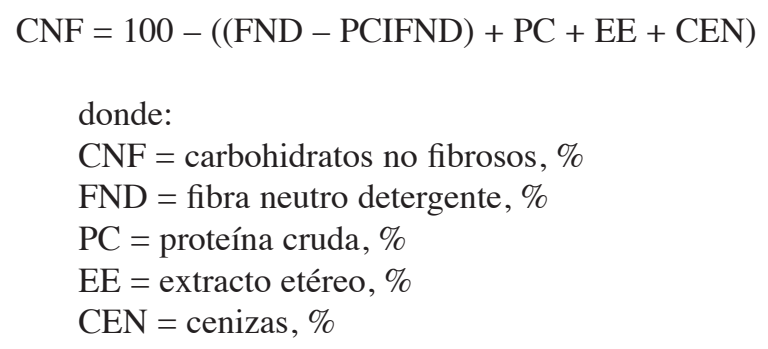

\section{Análisis de laboratorio}

Las muestras recolectadas fueron analizadas en el Laboratorio de Bromatología de la Estación Experimental "Alfredo Volio Mata" de la Universidad de Costa Rica, a cada una se le realizaron estudios de contenido de materia seca en una estufa a $105^{\circ} \mathrm{C}$, proteína cruda por el método de Kjeldahl, extracto etéreo y cenizas totales (AOAC, 2000). La fibra neutro detergente, la fibra ácido detergente y la lignina fueron procesadas según el método de Goering y Van-Soest (1970) y Van-Soest et al. (1991). Los valores de hemicelulosa y celulosa se estimaron por diferencia de las fracciones correspondientes. El contenido de proteína cruda incrustada en la fibra neutro detergente, se determinó efectuando primero la determinación de fibra neutro detergente (Goering y Van-Soest, 1970; Van-Soest et al., 1991), seguido por una determinación del contenido de proteína cruda presente en el residuo del análisis de fibra según el método de Kjeldahl (AOAC, 2000). El pH se determinó con un potenciómetro marca Daiger, modelo $6173 \mathrm{pH}$, serie JCO2826.

\section{Periodo experimental y análisis de resultados}

El periodo experimental tuvo una duración de 70 días, durante los cuales las bolsas con el ensilaje se mantuvieron almacenadas bajo techo y a temperatura ambiente; a estas se les realizó la recolección de efluentes cada siete días durante las primeras nueve semanas. Al finalizar dicho periodo, fueron pesadas y se abrieron para hacer una evaluación organoléptica (olor, color y textura) in situ. Se recolectó además una muestra del contenido de cada bolsa para efectuar los análisis de laboratorio.

Los valores de composición bromatológica del forraje inicial en mezcla con los aditivos, así como los contenidos nutricionales al finalizar el experimento fueron sometidos a análisis de varianza con el PROC ANOVA del paquete estadístico SAS Institute (1985). Aquellas variables que mostraron diferencias importantes entre medias, fueron sometidas a la prueba de Duncan para su separación estadística. Mediante este mismo procedimiento estadístico, se cuantificó la variación en las pérdidas ocurridas durante el proceso de fermentación del forraje. Por último, debido a que la cantidad de efluentes producida fue despreciable, no llegando a alcanzar niveles importantes su análisis fue excluido.

Con el fin de poder analizar las pérdidas de nutrientes generadas durante el proceso de fermentación del material, se estandarizó la cantidad de materia seca, los nutrientes y los componentes fibrosos en base a la cantidad presente en una bolsa de $50 \mathrm{~kg}$ de forraje verde con los aditivos agregados al inicio del experimento.

La evaluación organoléptica se efectuó inmediatamente luego de abrir las bolsas de ensilaje, 
según la metodología descrita por Betancourt et al. (2005) la cual considera los parámetros olor, color y textura como determinantes de la calidad del producto obtenido siguiendo la siguiente escala de medición:

- $\quad$ Escala de olor: 4 = agradable, 3 = ligeramente olor a moho, 2 = moderado olor a moho, 1 = fuerte olor a moho, 0 = fétido.

- Escala de color: 4 = verde pálido, 3 = verde musgo, 2 = verde oscuro, $1=$ verde pardo, $0=$ pardo.

- $\quad$ Escala de textura: 4 = firme y consistente, $3=$ consistente, $2=$ medio, $1=$ suave, $0=$ mucílago.

Donde:

$$
\begin{aligned}
& 4=\text { Excelente calidad } \\
& 3=\text { Buena calidad } \\
& 2=\text { Regular calidad } \\
& 1=\text { Mala calidad } \\
& 0=\text { Pésima calidad }
\end{aligned}
$$

\section{RESULTADOS Y DISCUSIÓN}

\section{Análisis bromatológicos}

La especie de pasto analizada presentó un porcentaje de proteína cruda bajo $(5,88 \%)$, un valor de fibra neutro detergente alto $(66,23 \%)$ y una concentración media de fibra ácido detergente $(40,76 \%)$ en base seca, esto en parte debido a la edad de rebrote permitida al forraje (Cuadro 1).

Existieron diferencias significativas $(p<0,05)$ según el tratamiento en cuanto al peso inicial del material en cada bolsa de ensilaje (Cuadro 2), siendo posible introducir una mayor cantidad de alimento en la bolsa al utilizar melaza como aditivo $(41,08$ $\mathrm{kg}$ ), y mínima cuando el forraje fue adicionado con Citrocom $(37,46 \mathrm{~kg})$. Se advierten también pérdidas de materia verde significativas $(\mathrm{p}<0,05)$ según el aditivo utilizado, hallando menores mermas cuando se empleó maíz molido con una reducción promedio de $11,68 \%$ y máximas variaciones en los tratamientos de melaza y pulpa de cítricos deshidratada los cuales contabilizaron reducciones promedio de $13,27 \%$ y $14,42 \%$ respectivamente. Por otro lado, no se determinaron diferencias importantes $(p>0,05)$ entre los niveles de cada tratamiento evaluado.

Existió una diferenciación significativa en la composición bromatológica del material a ensilar según el aditivo usado $(\mathrm{p}<0,05)$; mientras que no se detectaron cambios importantes $(p>0,05)$ entre los niveles de cada tratamiento. Se encontró un cambio en el nivel de materia seca según el tratamiento $(\mathrm{p}<0,05)$, siendo los parámetros mayores con el uso

\begin{tabular}{|c|c|c|c|c|}
\hline Componente nutricional & Ratana & Melaza & Maíz molido & Citrocom \\
\hline Materia seca (MS), $\%$ & 27,16 & 65,00 & 87,00 & 87,50 \\
\hline Proteína cruda (PC), \% & 5,88 & 3,00 & 8,00 & 10,00 \\
\hline Fibra neutro detergente (FND), $\%$ & 66,23 & -- & 12,50 & 43,17 \\
\hline Fibra ácido detergente (FAD), \% & 40,76 & -- & 2,99 & 17,53 \\
\hline Extracto etéreo (EE), \% & 2,17 & -- & 3,47 & 2,47 \\
\hline Cenizas totales (CEN), \% & 10,21 & 13,70 & 1,50 & 6,22 \\
\hline Lignina (LIG), \% & 4,54 & -- & 0,13 & 13,09 \\
\hline Celulosa (CEL), \% & 36,21 & -- & 2,86 & 4,45 \\
\hline Hemicelulosa (HEMI), \% & 25,72 & -- & 9,51 & 25,64 \\
\hline Proteína incrustada en la fibra neutro detergente (PCIFND), \% & -- & -- & 0,00 & 0,88 \\
\hline Carbohidratos no fibrosos (CNF), \% & 1,58 & -- & 74,53 & 39,02 \\
\hline Carbohidratos solubles (CHOS), \% & 17,09 & 34,00 & -- & -- \\
\hline
\end{tabular}
de maíz o Citrocom $(27,83 \%$ para ambos) que con el uso de melaza $(27,31 \%)$. Para todos los nutrientes se

Cuadro 1. Composición química del forraje de Ratana (Ischaemum indicum) y los aditivos usados para su conservación. Limón, Costa Rica. 2011. 
Cuadro 2. Peso inicial y final en base fresca del forraje de pasto Ratana (Ischaemum indicum) con aditivos para su conservación. Limón, Costa Rica. 2011.

\begin{tabular}{lccccc}
\hline Tratamiento & Nivel & $\begin{array}{c}\text { Peso inicial } \\
(\mathbf{k g})\end{array}$ & $\begin{array}{c}\text { Peso final } \\
(\mathbf{k g})\end{array}$ & $\begin{array}{c}\text { Diferencia de } \\
\text { peso }(\mathbf{k g})\end{array}$ & $\begin{array}{c}\text { Diferencia de } \\
\text { peso }(\mathbf{\%})\end{array}$ \\
\hline Melaza & 1 & 42,63 & 36,96 & 5,66 & 13,28 \\
& 2 & 40,13 & 34,66 & 5,46 & 13,61 \\
& 3 & 40,50 & 35,28 & 5,23 & 12,91 \\
Maíz molido & Prom & $\mathbf{4 1 , 0 8 a}$ & $\mathbf{3 5 , 6 3 a}$ & $\mathbf{5 , 4 5 a}$ & $\mathbf{1 3 , 2 7}$ \\
& & & & & \\
& 1 & 40,65 & 35,75 & 5,08 & 12,50 \\
& 2 & 39,63 & 35,74 & 4,90 & 12,36 \\
& 3 & 43,63 & 36,96 & 3,89 & 8,92 \\
Citrocom & Prom & $\mathbf{3 9 , 5 5 a b}$ & $\mathbf{3 4 , 9 3 a}$ & $\mathbf{4 , 6 2 b}$ & $\mathbf{1 1 , 6 8}$ \\
& 1 & & & & \\
& 2 & 38,50 & 32,85 & 5,65 & 14,68 \\
& 3 & 35,50 & 30,42 & 5,08 & 14,31 \\
& & 38,38 & 32,90 & 5,48 & 14,28 \\
& Prom & $\mathbf{3 7 , 4 6 b}$ & $\mathbf{3 2 , 0 6 b}$ & $\mathbf{5 , 4 0 a}$ & $\mathbf{1 4 , 4 2}$ \\
\hline
\end{tabular}

Letras diferentes en una columna, difieren estadísticamente $(\mathrm{p}<0,05)$.

encontraron variaciones significativas según el tipo de aditivo utilizado $(\mathrm{p}<0,05)$; aunque la variación real asociada a cada nutriente no llegó a alcanzar el $1,5 \%$. Los valores de proteína cruda del forraje inicial en mezcla con su respectivo nivel de aditivo, fueron significativamente diferentes entre sí para los tres tratamientos $(\mathrm{p}<0,05)$, donde el mayor porcentaje de proteína cruda se observó con el uso de maíz $(6,96 \%)$ y mínimo con el Citrocom (6,37\%); sin embargo, el residuo entre estos valores fue de únicamente $0,56 \%$. Para los componentes de la fibra, el extracto etéreo y las cenizas, se observó un comportamiento similar al ya mencionado para la proteína cruda, con diferencias pequeñas en números reales, pero estadísticamente significativas entre los tratamientos evaluados $(\mathrm{p}<0,05$; Cuadro 3).

Existieron diferencias significativas entre los tratamientos para las concentraciones de proteína cruda, celulosa y extracto etéreo $(p<0,05)$; manteniéndose en mayor porcentaje la proteína en el ensilaje con melaza $(6,44 \%)$, mientras que la celulosa y el extracto etéreo presentaron mayores proporciones en el ensilaje adicionado con Citrocom (40,63\% y $2,40 \%$, respectivamente). En el caso de los contenidos de materia seca, fibra neutro detergente, fibra ácido detergente, hemicelulosa, lignina y cenizas, no se determinaron diferencias significativas entre los tratamientos analizados ( $>00,05)$; esta ausencia de discrepancias en la composición del ensilaje final sugiere una uniformización cualitativa del ensilado al final del periodo experimental (70 días), con los tres tipos de aditivos e incluso, independientemente del nivel de aditivo agregado (Cuadro 4).

No se encontraron diferencias en la cantidad de nutrientes para cada nivel de inclusión de los diferentes aditivos ( $>0,05)$ (Cuadro 5); mientras que si se determinaron según el tratamiento utilizado $(p<0,05)$, siendo mayor la cantidad de materia seca cuando se mezcló el forraje con maíz molido $(13,92 \mathrm{~kg})$ o Citrocom $(13,91 \mathrm{~kg})$ que cuando se utilizó melaza como agregado $(13,66 \mathrm{~kg})$. El comportamiento de la proteína cruda también presentó diferencias según el aditivo empleado, siendo mayor la concentración del componente cuando se usó maíz molido $(0,968$ $\mathrm{kg})$, media con la melaza $(0,935 \mathrm{~kg})$ y baja con el Citrocom $(0,887 \mathrm{~kg})$. Similar conducta se observó para los distintos componentes de la fibra, el extracto etéreo y las cenizas los cuales mostraron diferencias significativas entre tratamientos $(p<0,05)$ no así entre niveles de inclusión del aditivo ( $p>0,05)$; la fibra neutro detergente y la fibra ácido detergente fueron máximas con el Citrocom, de valor medio con la 
Cuadro 3. Composición nutricional inicial del forraje de pasto Ratana (Ischaemum indicum) con su correspondiente nivel de aditivo en \%. Limón, Costa Rica. 2011.

\begin{tabular}{|c|c|c|c|c|c|c|c|c|c|c|}
\hline Tratamiento & Nivel & MS & PC & FND & FAD & HEMI & CEL & LIG & $\mathbf{E E}$ & CEN \\
\hline \multirow[t]{4}{*}{ Melaza } & 1 & 27,16 & 6,88 & 66,23 & 40,76 & 25,47 & 36,20 & 4,54 & 2,17 & 10,21 \\
\hline & 2 & 27,31 & 6,85 & 65,50 & 40,31 & 25,19 & 35,80 & 4,49 & 2,14 & 10,25 \\
\hline & 3 & 27,46 & 6,82 & 64,80 & 39,88 & 24,93 & 35,40 & 4,45 & 2,12 & 10,29 \\
\hline & Prom & 27,31b & $6,85 b$ & $65,51 \mathrm{a}$ & $40,32 a$ & $25,20 \mathrm{~b}$ & $35,85 a$ & $4,49 \mathrm{a}$ & $2,14 c$ & $10,25 a$ \\
\hline \multirow[t]{4}{*}{ Maíz molido } & 1 & 27,60 & 6,93 & 65,00 & 39,75 & 25,05 & 35,33 & 4,43 & 2,20 & 9,98 \\
\hline & 2 & 27,82 & 6,96 & 64,13 & 39,28 & 24,85 & 34,91 & 4,37 & 2,22 & 9,86 \\
\hline & 3 & 28,07 & 6,99 & 63,49 & 38,84 & 24,66 & 34,52 & 4,32 & 2,24 & 9,77 \\
\hline & Prom & $27,83 a$ & $6,96 a$ & $64,14 \mathrm{c}$ & $39,29 \mathrm{c}$ & $24,85 c$ & $34,92 \mathrm{c}$ & $4,37 \mathrm{~b}$ & $2,22 \mathrm{a}$ & $9,88 \mathrm{c}$ \\
\hline \multirow[t]{4}{*}{ Citrocom } & 1 & 27,61 & 6,99 & 65,61 & 40,14 & 25,48 & 35,37 & 4,42 & 2,18 & 10,11 \\
\hline & 2 & 27,84 & 6,04 & 65,31 & 39,83 & 25,48 & 34,94 & 4,36 & 2,18 & 10,05 \\
\hline & 3 & 28,04 & 6,09 & 65,03 & 39,55 & 25,48 & 35,25 & 4,31 & 2,18 & 10,00 \\
\hline & Prom & $27,83 a$ & $6,37 c$ & $65,32 b$ & $39,84 b$ & $25,48 a$ & $35,18 b$ & $4,36 c$ & $2,18 b$ & $10,05 b$ \\
\hline
\end{tabular}

$\mathrm{MS}=$ materia seca, $\mathrm{PC}=$ proteína cruda, $\mathrm{FND}=$ fibra neutro detergente, $\mathrm{FAD}=$ fibra ácido detergente, $\mathrm{HEMI}=$ hemicelulosa, $\mathrm{CEL}=$ celulosa, $\mathrm{LIG}=$ lignina, $\mathrm{EE}=$ extracto etéreo, $\mathrm{CEN}=$ cenizas .

Letras diferentes en una columna, difieren estadísticamente $(\mathrm{p}<0,05)$.

Cuadro 4. Composición nutricional final del forraje de pasto Ratana (Ischaemum indicum) ensilado con su correspondiente nivel de aditivo, en \%. Limón, Costa Rica. 2011.

\begin{tabular}{|c|c|c|c|c|c|c|c|c|c|c|}
\hline Tratamiento & Nivel & MS & PC & FND & FAD & HEMI & CEL & LIG & $\mathbf{E E}$ & CEN \\
\hline \multirow[t]{4}{*}{ Melaza } & 1 & 28,77 & 6,30 & 68,80 & 42,15 & 26,67 & 37,28 & 4,87 & 2,17 & 10,33 \\
\hline & 2 & 27,99 & 6,47 & 68,80 & 43,93 & 24,84 & 38,88 & 5,04 & 2,21 & 10,51 \\
\hline & 3 & 26,87 & 6,55 & 68,30 & 44,79 & 23,49 & 39,74 & 5,05 & 2,28 & 10,21 \\
\hline & Prom & 27,86 & $6,44 a$ & 68,63 & 43,62 & 25,00 & $38,63 b$ & 4,99 & $2,22 \mathrm{~b}$ & 10,35 \\
\hline \multirow[t]{4}{*}{ Maíz molido } & 1 & 30,20 & 6,12 & 66,65 & 41,52 & 25,14 & 36,91 & 4,61 & 2,22 & 10,39 \\
\hline & 2 & 23,28 & 6,38 & 71,08 & 49,67 & 21,41 & 44,45 & 5,22 & 2,52 & 11,02 \\
\hline & 3 & 26,61 & 6,07 & 68,89 & 44,43 & 24,46 & 39,57 & 4,86 & 2,30 & 10,28 \\
\hline & Prom & 26,88 & 6,19ab & 68,87 & 45,20 & 23,67 & 40,31ab & 4,89 & 2,35ab & 10,56 \\
\hline \multirow[t]{4}{*}{ Citrocom } & 1 & 24,37 & 5,97 & 71,82 & 48,20 & 23,61 & 43,08 & 5,13 & 2,56 & 10,32 \\
\hline & 2 & 28,51 & 5,85 & 69,68 & 45,05 & 24,64 & 40,15 & 4,90 & 2,30 & 9,93 \\
\hline & 3 & 27,77 & 6,12 & 68,25 & 43,52 & 24,73 & 38,67 & 4,84 & 2,36 & 10,54 \\
\hline & Prom & 26,70 & $5,98 b$ & 69,91 & 45,59 & 24,33 & $40,63 a$ & 4,96 & $2,40 \mathrm{a}$ & 10,26 \\
\hline
\end{tabular}

$\mathrm{MS}=$ materia seca, $\mathrm{PC}=$ proteína cruda, $\mathrm{FND}=$ fibra neutro detergente, $\mathrm{FAD}=$ fibra ácido detergente, $\mathrm{HEMI}=$ hemicelulosa, $\mathrm{CEL}=$ celulosa, $\mathrm{LIG}=$ lignina, $\mathrm{EE}=$ extracto etéreo, $\mathrm{CEN}=$ cenizas .

Letras diferentes en una columna, difieren estadísticamente $(\mathrm{p}<0,05)$. 
Cuadro 5. Cantidad de nutrientes iniciales en $\mathrm{kg}$ por cada $50 \mathrm{~kg}$ de forraje fresco de pasto Ratana (Ischaemum indicum) con diferentes niveles de aditivo. Limón, Costa Rica. 2011.

\begin{tabular}{|c|c|c|c|c|c|c|c|c|c|c|}
\hline Tratamiento & Nivel & MS & PC & FND & FAD & HEMI & CEL & LIG & EE & CEN \\
\hline \multirow[t]{4}{*}{ Melaza } & 1 & 13,58 & 0,934 & 8,994 & 5,535 & 3,459 & 4,918 & 0,617 & 0,294 & 1,387 \\
\hline & 2 & 13,66 & 0,935 & 8,945 & 5,504 & 3,440 & 4,891 & 0,614 & 0,293 & 1,339 \\
\hline & 3 & 13,73 & 0,936 & 8,898 & 5,476 & 3,442 & 4,865 & 0,610 & 0,291 & 1,413 \\
\hline & Prom & $13,66 b$ & $0,935 b$ & $8,946 b$ & $5,505 b$ & $3,440 \mathrm{c}$ & $4,891 \mathrm{a}$ & $0,614 a$ & $0,293 \mathrm{c}$ & $1,400 \mathrm{a}$ \\
\hline \multirow[t]{4}{*}{ Maíz molido } & 1 & 13,80 & 0,957 & 8,943 & 5,486 & 3,457 & 4,875 & 0,611 & 0,304 & 1,377 \\
\hline & 2 & 13,91 & 0,968 & 8,922 & 5,464 & 3,457 & 4,857 & 0,608 & 0,309 & 1,373 \\
\hline & 3 & 14,04 & 0,981 & 8,911 & 5,450 & 3,461 & 4,844 & 0,606 & 0,314 & 1,371 \\
\hline & Prom & $13,92 a$ & $0,968 \mathbf{a}$ & $8,925 c$ & $5,467 \mathrm{c}$ & $3,458 b$ & $4,858 b$ & $0,608 b$ & $0,308 \mathbf{a}$ & $1,374 b$ \\
\hline \multirow[t]{4}{*}{ Citrocom } & 1 & 13,80 & 0,964 & 9,056 & 5,539 & 3,516 & 4,881 & 0,610 & 0,300 & 1,395 \\
\hline & 2 & 13,92 & 0,841 & 9,089 & 5,543 & 3,546 & 4,863 & 0,607 & 0,303 & 1,399 \\
\hline & 3 & 14,02 & 0,854 & 9,119 & 5,546 & 3,573 & 4,942 & 0,604 & 0,306 & 1,403 \\
\hline & Prom & $13,91 a$ & $0,887 \mathrm{c}$ & 9,088a & 5,543a & 3,545a & 4,895a & $0,607 \mathrm{c}$ & $0,303 b$ & 1,399a \\
\hline
\end{tabular}

$\mathrm{MS}=$ materia seca, $\mathrm{PC}=$ proteína cruda, $\mathrm{FND}=$ fibra neutro detergente, $\mathrm{FAD}=$ fibra ácido detergente, $\mathrm{HEMI}=$ hemicelulosa, $\mathrm{CEL}=$ celulosa, $\mathrm{LIG}=$ lignina, $\mathrm{EE}=$ extracto etéreo, $\mathrm{CEN}=$ cenizas.

$\mathrm{a}, \mathrm{b}, \mathrm{c}$ marcan diferencias estadísticas entre columnas $(\mathrm{p}<0,05)$.

melaza y mínimos con el uso de maíz molido como tratamiento; la hemicelulosa presentó niveles máximos en las muestras que incluían maíz, y fue mínima con la melaza; la celulosa y las cenizas se comportaron de igual manera, presentando cantidades estadísticamente similares con el uso de melaza y Citrocom, siendo estos mayores que cuando se utilizó maíz molido como aditivo; la concentración de lignina fue menor cuando se usó Citrocom en vez de maíz y esta a su vez fue más reducida que con el uso de melaza; por último, el maíz potenció la concentración de extracto etéreo en el material haciendo que se presentaran los valores más altos de dicho componente, mientras que el tratamiento que incluía melaza como agregado presentó los tenores más bajos observados.

Los datos sobre la cantidad de nutrientes en el ensilaje recolectados luego del periodo de fermentación (Cuadro 6), no muestran diferencias significativas en los distintos componentes del material, tanto entre tratamientos como niveles de inclusión del aditivo $(p>0,05)$ excepto, para los contenidos de proteína cruda y lignina, los cuales si presentaron variaciones importantes según el tratamiento $(p<0,05)$. Para el caso de la proteína, el valor fue máximo con el uso de melaza $(0,774 \mathrm{~kg} /$ bolsa de $50 \mathrm{~kg})$ y mínimo con la mezcla del forraje con el Citrocom $(0,682 \mathrm{~kg} /$ bolsa de $50 \mathrm{~kg}$ ); por su parte, la lignina se encontró en concentraciones estadísticamente similares en los procedimientos de ensilaje que incluían melaza $(0,598$ $\mathrm{kg} / \mathrm{bolsa}$ de $50 \mathrm{~kg})$ y Citrocom $(0,599 \mathrm{~kg} /$ bolsa de 50 $\mathrm{kg}$ ), siendo esas cantidades mayores a lo encontrado con maíz molido $(0,567 \mathrm{~kg} /$ bolsa de $50 \mathrm{~kg})(\mathrm{p}<0,05)$.

En el Cuadro 7 se muestran las pérdidas de materia seca y los componentes nutricionales en los ensilajes elaborados en términos porcentuales, observándose únicamente diferencias importantes entre tratamientos para los contenidos de proteína, hemicelulosa y lignina $(\mathrm{p}<0,05)$. La mayor reducción en la cantidad de proteína se dio al utilizar maíz molido, al producir este aditivo una reducción de $0,248 \mathrm{~kg}$ de $\mathrm{PC} / 50 \mathrm{~kg}$ de forraje ensilado contra 0,205 y $0,161 \mathrm{~kg}$ de PC/50 kg de forraje del Citrocom y la melaza, respectivamente. Por 
Cuadro 6. Cantidad de nutrientes finales en $\mathrm{kg}$ por cada $50 \mathrm{~kg}$ de forraje de pasto Ratana (Ischaemum indicum) ensilado con diferentes niveles de aditivo. Limón, Costa Rica. 2011.

\begin{tabular}{|c|c|c|c|c|c|c|c|c|c|c|}
\hline Tratamiento & Nivel & MS & PC & FND & FAD & HEMI & CEL & LIG & EE & CEN \\
\hline \multirow[t]{4}{*}{ Melaza } & 1 & 12,45 & 0,785 & 8,563 & 5,247 & 3,316 & 4,642 & 0,606 & 0,270 & 1,286 \\
\hline & 2 & 12,04 & 0,778 & 8,269 & 5,274 & 2,994 & 4,670 & 0,605 & 0,265 & 1,264 \\
\hline & 3 & 11,61 & 0,759 & 7,914 & 5,182 & 2,733 & 4,599 & 0,583 & 0,264 & 1,184 \\
\hline & Prom & 12,03 & $0,774 a$ & 8,249 & 5,234 & 3,014 & 4,637 & $0,598 \mathrm{a}$ & 0,266 & 1,245 \\
\hline \multirow[t]{4}{*}{ Maíz molido } & 1 & 12,99 & 0,795 & 8,660 & 5,394 & 3,266 & 4,795 & 0,599 & 0,289 & 1,349 \\
\hline & 2 & 10,13 & 0,646 & 7,204 & 5,103 & 2,191 & 4,484 & 0,529 & 0,256 & 1,115 \\
\hline & 3 & 11,82 & 0,723 & 8,118 & 5,209 & 2,909 & 4,637 & 0,571 & 0,271 & 1,213 \\
\hline & Prom & 11,65 & $0,721 \mathrm{ab}$ & 7,994 & 5,205 & 2,789 & 4,639 & $0,567 b$ & 0,272 & 1,226 \\
\hline \multirow[t]{4}{*}{ Citrocom } & 1 & 10,31 & 0,619 & 7,402 & 4,965 & 2,437 & 4,448 & 0,527 & 0,263 & 1,066 \\
\hline & 2 & 12,07 & 0,706 & 8,391 & 5,409 & 2,982 & 4,820 & 0,589 & 0,276 & 1,197 \\
\hline & 3 & 11,71 & 0,720 & 7,956 & 5,070 & 2,887 & 4,509 & 0,501 & 0,275 & 1,230 \\
\hline & Prom & 11,37 & $0,682 b$ & 7,917 & 5,148 & 2,769 & 4,589 & $0,599 b$ & 0,271 & 1,164 \\
\hline
\end{tabular}

$\mathrm{MS}=$ materia seca, $\mathrm{PC}=$ proteína cruda, $\mathrm{FND}=$ fibra neutro detergente, $\mathrm{FAD}=$ fibra ácido detergente, $\mathrm{HEMI}=$ hemicelulosa, $\mathrm{CEL}=$ celulosa, $\mathrm{LIG}=$ lignina, $\mathrm{EE}=$ extracto etéreo, $\mathrm{CEN}=$ cenizas. a, b marcan diferencias estadísticas entre columnas $(\mathrm{p}<0,05)$.

Cuadro 7. Contenido porcentual de nutrientes perdidos en el forraje de pasto Ratana (Ischaemum indicum) ensilado tratado con diferentes niveles de aditivos. Limón, Costa Rica. 2011.

\begin{tabular}{|c|c|c|c|c|c|c|c|c|c|c|}
\hline Tratamiento & Nivel & MS & PC & FND & FAD & HEMI & CEL & LIG & EE & CEN \\
\hline \multirow[t]{4}{*}{ Melaza } & 1 & 8,34 & 15,95 & 4,79 & 5,20 & 4,13 & 5,61 & 1,94 & 8,16 & 7,28 \\
\hline & 2 & 11,84 & 16,79 & 7,57 & 4,18 & 12,99 & 4,54 & 1,47 & 9,56 & 10,16 \\
\hline & 3 & 15,49 & 19,02 & 11,06 & 5,37 & 20,16 & 5,49 & 4,43 & 9,62 & 16,21 \\
\hline & Prom & 11,89 & $17,25 b$ & 7,81 & 4,92 & $12,43 b$ & 5,21 & $2,61 b$ & 9,11 & 11,22 \\
\hline \multirow[t]{4}{*}{ Maíz molido } & 1 & 5,83 & 17,03 & 3,16 & 1,68 & 5,53 & 1,64 & 1,96 & 4,93 & 2,11 \\
\hline & 2 & 27,15 & 33,37 & 19,24 & 8,27 & 36,62 & 7,68 & 12,99 & 16,83 & 18,86 \\
\hline & 3 & 15,80 & 26,30 & 8,91 & 4,44 & 15,95 & 4,27 & 5,78 & 13,69 & 11,52 \\
\hline & Prom & 16,26 & $25,57 a$ & 10,44 & 4,80 & 19,37ab & 4,53 & $6,91 \mathrm{ab}$ & 11,82 & 10,83 \\
\hline \multirow[t]{4}{*}{ Citrocom } & 1 & 25,28 & 35,79 & 18,25 & 10,38 & 30,69 & 9,10 & 13,61 & 12,67 & 23,58 \\
\hline & 2 & 13,25 & 16,05 & 7,68 & 2,42 & 15,91 & 0,88 & 2,97 & 9,24 & 14,44 \\
\hline & 3 & 16,49 & 15,81 & 12,75 & 8,58 & 19,20 & 8,76 & 7,12 & 10,46 & 12,33 \\
\hline & Prom & 18,34 & $22,55 \mathrm{ab}$ & 12,90 & 7,13 & 21,93a & 6,25 & $7,90 \mathrm{a}$ & 10,79 & 16,78 \\
\hline
\end{tabular}

$\mathrm{MS}=$ materia seca, $\mathrm{PC}=$ proteína cruda, $\mathrm{FND}=$ fibra neutro detergente, $\mathrm{FAD}=$ fibra ácido detergente, $\mathrm{HEMI}=$ hemicelulosa, $\mathrm{CEL}=$ celulosa, $\mathrm{LIG}=$ lignina, $\mathrm{EE}=$ extracto etéreo, $\mathrm{CEN}=$ cenizas.

a, b marcan diferencias estadísticas entre columnas $(\mathrm{p}<0,05)$. 
su parte, la hemicelulosa se vio reducida $0,776 \mathrm{~kg} / 50$ $\mathrm{kg}$ de forraje al utilizar Citrocom como agregado, 0,669 $\mathrm{kg} / 50 \mathrm{~kg}$ de forraje al usar maíz y $0,426 \mathrm{~kg} / 50 \mathrm{~kg}$ de forraje con melaza; la lignina disminuyó 0,$048 ; 0,042$ y $0,016 \mathrm{~kg} / 50 \mathrm{~kg}$ de forraje cuando los aditivos fueron Citrocom, maíz y melaza respectivamente, haciendo que la melaza produjera el menor cambio y el Citrocom el mayor en dichos componentes fibrosos.

\section{Características organolépticas y producción de efluentes}

Para los valores de $\mathrm{pH}$, no se encontró variación significativa entre los niveles de aditivo agregado $(\mathrm{p}>0,05)$, aunque si la hubo entre tratamientos $(\mathrm{p}<0,05)$, siendo estadísticamente menor el $\mathrm{pH}$ medido cuando se utilizó melaza $(4,84)$ que cuando se utilizó Citrocom o maíz (5,02 y 5,10 respectivamente). En cuanto a las propiedades organolépticas observadas, la adición de maíz molido y Citrocom generó un olor agradable en todos los niveles de inclusión empleados, produciéndose apreciaciones similares con el uso de melaza, la cual presentó un poco de crecimiento de hongos en el nivel menor de inclusión de aditivo. El color, en general se observó como un verde pálido en todos los tratamientos que incluyeron maíz molido, similares anotaciones generó el uso de Citrocom el cual presentó un color verde pálido para los niveles de $1 \%$ y $3 \%$ de inclusión y un ligero color musgoso cuando el porcentaje de inclusión fue de 2 . Cuando se evaluó el tratamiento de la melaza con un $2 \%$ de inclusión del aditivo, el color observado fue verde pálido mientras que en los otros dos niveles evaluados, el color se acercó más al verde musgo. Por último, todos los tratamientos evaluados produjeron ensilajes con una textura firme y consistente, característica buscada en un ensilaje de buena calidad (Cuadro 8).

\section{Evaluación de la composición química del pasto Ratana}

El valor de MS en pasto Ratana de $22,8 \%$, obtenido por Sánchez et al. (1998), es menor al encontrado en este trabajo; debido probablemente al menor tiempo de rebrote permitido en dicha investigación (máximo veinticinco días) y la mayor precipitación promedio en el presente trabajo ( $2815 \mathrm{~mm}$ anuales contra $4577,2 \mathrm{~mm}$ anuales) la cual estimuló el crecimiento de la pastura. Por otro lado, a pesar de que el forraje en este experimento se cosechó con 70 días de crecimiento, presentó contenidos de MS inferiores a los recomendados por otros autores para la elaboración de ensilajes los cuales

Cuadro 8. Características organolépticas del ensilado de pasto Ratana (Ischaemum indicum) con los diferentes aditivos estudiados. Limón, Costa Rica. 2011.

\begin{tabular}{|c|c|c|c|c|c|}
\hline \multirow[b]{2}{*}{ Tratamiento } & \multirow[b]{2}{*}{ Nivel } & \multirow[b]{2}{*}{ pH } & \multicolumn{3}{|c|}{ Puntaje } \\
\hline & & & Olor & Color & Textura \\
\hline \multirow[t]{4}{*}{ Melaza } & 1 & 4,85 & 3,5 & 3,25 & 4 \\
\hline & 2 & 4,87 & 4 & 4 & 4 \\
\hline & 3 & 4,80 & 4 & 3,25 & 4 \\
\hline & Prom & $4,84 \mathrm{~b}$ & -- & -- & -- \\
\hline \multirow[t]{4}{*}{ Maíz } & 1 & 5,25 & 4 & 4 & 4 \\
\hline & 2 & 5,14 & 4 & 4 & 4 \\
\hline & 3 & 4,92 & 4 & 4 & 4 \\
\hline & Prom & $5,10 \mathrm{a}$ & -- & & -- \\
\hline \multirow[t]{4}{*}{ Citrocom } & 1 & 4,94 & 4 & 4 & 4 \\
\hline & 2 & 5,06 & 4 & 3,75 & 4 \\
\hline & 3 & 5,10 & 4 & 4 & 4 \\
\hline & Prom & $5,02 \mathrm{a}$ & -- & -- & -- \\
\hline
\end{tabular}

a, b marcan diferencias estadísticas entre columnas $(\mathrm{p}<0,05)$. 
indican un mínimo necesario de 30-35\% (Santos et al., 2010), ya que niveles inferiores producen pérdidas de nutrientes durante el proceso de conservación, debido a la formación de efluentes o fermentaciones indeseables (Bergamaschine et al., 2006).

Valores promedio de PC de $9,1 \%$ con materiales de 21 a 25 días de desarrollo fueron indicados por Sánchez y Soto (1999), mientras que Ng (1972) reportó valores promedio de $7,91 \%$, siendo estos resultados mayores al 5,88\% encontrado; diferencia que puede explicarse debido al tiempo de rebrote, o a la ausencia de fertilización nitrogenada del suelo, ya que Villareal (2010) determinó tenores de hasta 17,5\% de proteína en pasturas de Ratana, fertilizadas con dosis de $450 \mathrm{~kg}$ de N/ha/año. Además, debe tomarse en consideración que el porcentaje de $\mathrm{PC}$ promedio encontrado no alcanza el 7\% mínimo recomendado para que el consumo, y la digestibilidad de la materia seca del forraje sean óptimos (Mazza et al., 2007).

Los datos de FND determinados $(66,23 \%$ en base seca) son similares a los reportados por Sánchez y Soto (1999) y por Sánchez et al. (1998), quienes encontraron niveles de $68,8 \%$ y $69,1 \%$ respectivamente. Según Villareal (2010), la especie Ischaemum indicum Houtt. con fertilización nitrogenada puede exhibir valores de $37,4 \%$ de FAD durante la época lluviosa, lo cual no dista mucho de lo encontrado en el presente estudio.

\section{Evaluación de la materia seca del ensilaje}

Porcentajes de MS de 17,17\% y 16,9\% fueron encontrados por Nisa et al. (2008) al utilizar maíz en ensilajes de Jambo grass (Sorghum bicolor x Sorghum sudanefe), en porcentajes de $2 \%$ y $4 \%$ respectivamente; en el mismo estudio, con el uso de melaza se presentan niveles de MS cercanos al 18\% usando la misma proporción de aditivo mencionada; los cuales son menores a los encontrados con los mismos grados de inclusión en el pasto Ratana, probablemente debido al contenido de materia seca inicial de los forrajes (18,0\% contra $27,16 \%$ del presente estudio). En el caso del Citrocom como aditivo, los valores de MS para Pennisetum purpureum, Schum de $21,37 \%$ y 23,92\% son presentados por Mazza et al. (2005) para inclusiones de $2,5 \%$ y $5 \%$ respectivamente; porcentajes que son más bajos pero cercanos a los obtenidos con los niveles evaluados.

Disminuciones en el porcentaje de MS en ensilajes de Pennisetum purpureum cv. Mott adicionado con maíz molido o melaza en diferentes niveles fueron obtenidas por Qamar (2009), situación que difiere a lo encontrado ya que, aunque si existieron diferencias entre los aditivos analizados y las diferentes proporciones de los mismos, estos no presentaron variaciones significativas. Según Mazza et al. (2005), el uso de la pulpa de cítricos como aditivo puede generar pérdidas de material durante el proceso de fermentación debido al aumento en la disponibilidad de carbohidratos solubles para los microorganismos al generarse pérdidas de carbono en forma de $\mathrm{CO}_{2} \mathrm{O}$ productos de naturaleza volátil como ácidos orgánicos o etanol; situación que podría explicar las pérdidas de forraje encontradas en el experimento realizado.

\section{Evaluación de la proteína cruda del ensilaje}

En ensilajes elaborados con Jambo grass, Nisa et al. (2008) no encontraron diferencias en la PC, ni con el uso de melaza o maíz molido, ni entre la cantidad de agregado utilizada, situación diferente a lo encontrado en este estudio, ya que sí se determinaron variaciones significativas en el uso de melaza o maíz $(p<0,05)$. En otro estudio, Huisden et al. (2009) encontraron valores de PC de 6,51\% de la materia seca en ensilaje de maíz adicionado con melaza, valor cercano al encontrado para el pasto Ratana; mientras que ensilando $P$. purpureum cv. Mott con maíz molido como aditivo (3\% de agregado), Qamar (2009) determinó una concentración de PC de $14,15 \%$, la cual es muy superior a la determinada en el tratamiento con este aditivo.

En ensilaje de pasto Elefante (P. purpuerum, Schum) el nivel de proteína promedio fue de $8,91 \%$ usando citropulpa (0, 3, 6 o 9\% de inclusión) (Mazza et al., 2007), valor superior al obtenido en este estudio, debido a la cantidad de proteína inicial en el pasto utilizado para el estudio. También diferente a lo encontrado en esta investigación, Mazza et al. (2005) determinaron un efecto cuadrático significativo para el nivel de inclusión de pulpa de cítricos deshidratada en el ensilaje de pasto Elefante al analizar porcentajes desde $0 \%$ hasta $15 \%$, con un máximo al utilizar un $7,61 \%$ de Citrocom, aunque esta diferencia se puede deber a los niveles de aditivo utilizados en este estudio los cuales fueron menores.

Araujo-Febres et al. (1996) consideran como normal pérdidas de hasta $60 \%$ de la PC durante el proceso de ensilaje a causa de que el nivel de $\mathrm{pH}$ 
alcanzado no es lo suficientemente bajo para detener la actividad de enzimas proteolíticas en la planta, ya que estas se inactivan completamente a niveles de 3,8 a 4,5 (Qamar, 2009), lo cual conlleva a una mayor disminución de la PC en el ensilaje. Además, la cantidad de bacterias desarrolladas durante el proceso de fermentación podría no ser suficiente para equilibrar las pérdidas de proteína generadas durante la conservación del pasto (Qamar, 2009).

\section{Evaluación de la fibra y sus componentes en el ensilaje}

En el presente ensayo no se encontraron variaciones significativas para la concentración de FND o FAD con los aditivos utilizados en los ensilajes, ni entre los porcentajes agregados en los tratamientos; mientras que en un ensayo realizado por Nisa et al. (2008) ensilando Jambo grass con melaza o maíz molido (2\%, $4 \%$ y $6 \%$ de inclusión), si se determinaron diferencias significativas entre aditivos para la concentración de FND del ensilaje debido probablemente a la diferencia en la composición nutricional inicial entre los forrajes. En dicha investigación, además la FAD no presentó ninguna variación estadística similar a lo encontrado con el pasto Ratana.

Valores muy similares a los reportados en la literatura fueron encontrados en el presente estudio para la FND y FAD; Araujo-Febres et al. (1996) determinaron concentraciones de $60,24 \%$ y $40,21 \%$ para la FND y FAD respectivamente, para ensilajes de pasto elefante enano $(P$. purpureum $\mathrm{cv}$. Mott). Nisa et al. (2008) determinaron tenores de FND de alrededor de $70 \%$ y de FAD de $42 \%$ utilizando maíz molido en inclusiones de $2 \%$ y $4 \%$ en ensilajes de Jambo grass (Sorghum bicolor $x$ Sorghum sudanefe). En el caso del uso de Citrocom como aditivo (5\% de inclusión) en ensilajes de pasto Camerún (P. purpureum Schum) se reportan valores de FAD y FND de 47,78\% y $67,38 \%$ respectivamente (Pereira et al., 1999).

En el trabajo realizado por Nisa et al. (2008), se determinaron diferencias significativas entre el uso de maíz molido o melaza y los niveles de agregado para la hemicelulosa, mientras que para la celulosa y la lignina no se encontró ningún efecto entre los tratamientos evaluados, situación que difiere levemente a lo encontrado con el pasto Ratana para el cuál únicamente se encontró variaciones en la celulosa según el aditivo empleado. Similar a lo medido en este trabajo, López et al. (2009) no se encontraron variaciones con importancia estadística al ensilar rastrojos de piña con niveles crecientes de pulpa de cítricos deshidratada.

En análisis realizados a ensilaje de maíz adicionado con melaza, Huisden et al. (2009) encontraron valores de hemicelulosa de $21,8 \%$ los cuales son menores a lo apreciado, este contenido menor puede deberse a la composición nutricional del maíz, o a degradaciones indeseables durante el proceso de fermentación (Bolsen et al., 1996); situación similar se observó cuando se utilizó melaza, aditivos microbiales y niveles crecientes de pulpa de cítricos deshidratada al presentar valores que rondan el $15 \%$ de la materia seca (López et al., 2009). Cuando se usó un 6\% de maíz molido como aditivo del ensilaje, Nisa et al. (2008) determinaron contenidos de hemicelulosa de $25,97 \%$ valor muy similar al obtenido en el presente estudio con pasto Ratana.

La literatura reporta valores de celulosa cuando la inclusión de aditivo fue de $4 \%$ de $38,8 \%$ y $39,2 \%$ para el uso maíz molido o melaza respectivamente, en ensilajes de un híbrido de Sorghum bicolor $x$ Sorghum sudanefe (Nisa et al., 2008); y de 37,55\% en ensilajes de Pannicum máximum Jacq. cv. Tanzania I con un 5\% de pulpa de cítricos (Botega et al., 2009) concentraciones bastante similares a las encontradas en el ensayo realizado.

El empleo de un $4 \%$ de maíz molido en ensilaje de sorgo (Nisa et al., 2008) mostró cantidades de lignina de 3,63\% de la materia seca, mientras que Araujo-Febres et al. (1996) encontraron contenidos de lignina de $6,71 \%$ en silos de $P$. purpureum cv. Mott cosechado a 63 días de rebrote y adicionado con un $4 \%$ de melaza; por último Botega et al. (2009), indicaron tenores de 5,08\% para el componente en pasto Tanzania I con 5\% de citropulpa. Los valores similares de los diferentes componentes de la fibra podrían indicar una estabilización del ensilaje al final del proceso de fermentación independientemente del forraje utilizado.

Las disminuciones en la fibra se produjeron probablemente a causa de una falta de carbohidratos solubles, lo cual llevó a las bacterias productoras de ácido láctico a fermentar azúcares provenientes de la ruptura de la hemicelulosa en la pared celular (Bolsen et al., 1996). Según Pereira et al. (1999) 
esta disminución de la FDN y FDA son deseables en ensilajes de buena calidad, ya que disminuye el tiempo necesario para la digestión del alimento en el retículo-rumen del animal, aumentando la tasa de pasaje del alimento en el tracto digestivo y por lo tanto, aumentando el consumo de materia seca.

\section{Evaluación del extracto etéreo en el ensilaje}

Los valores de extracto etéreo encontrados están sobre el mínimo normal de $2,1 \%$ reportado por Santos et al. (2010), para ensilajes de buena calidad elaborados con forrajeras tropicales; la composición encontrada es también similar a la reportada para el componente por Bergamaschine et al. (2006), para ensilajes adicionados con pulpa de cítricos.

\section{Evaluación de las cenizas en el ensilaje}

Al usar maíz molido en niveles de 3\%, Qamar (2009) encontró concentraciones de cenizas de 11,15\% en ensilajes elaborados con $P$. purpureum cv. Mott como forraje, mientras que al ensilar el mismo pasto pero usando melaza como aditivo (3\% de la materia seca) encontró tenores de $11,34 \%$, similares a las determinadas para el pasto Ratana. Por otra parte, Ferrari-Junior et al. (2009) reportan porcentajes de $7,81 \%$ ensilando Pennisetm hybridum cv. Paraíso con un $5 \%$ de pulpa de cítricos deshidratada.

\section{Evaluación organoléptica del ensilaje}

Similar a lo señalado en este estudio, Qamar (2009) determinó una diferencia estadísticamente significativa entre el uso de melaza o maíz como aditivo sobre el pH en ensilajes de pasto elefante enano, en proporciones de hasta 5\% de inclusión de ambos agregados, aunque sus observaciones difieren de lo encontrado en el nivel utilizado de cada complemento, ya que dicho autor sí determinó una disminución de importancia estadística al aumentar el porcentaje de inclusión de ambos materiales. Por otro lado, en el estudio realizado por Nisa et al. (2008) utilizando también maíz molido y melaza al ensilar sorgo, no se encontraron variaciones importantes ni entre aditivos ni los niveles incluidos en el valor de $\mathrm{pH}$ observado.

La literatura reporta valores de $\mathrm{pH}$ de 4,56 en ensilajes de caña de azúcar y rabo de ratón (Equisetum arvense) con un 4\% de melaza (Suárez et al., 2011); 3,80 en ensilaje de sorgo con maíz molido ( $4 \%$ de inclusión) (Nisa et al., 2008); y 4,17 al ensilar $B$. brizantha cv. Marandú con un 10\% de pulpa de cítricos peletizada; siendo todos estos valores inferiores a lo determinado, además de que la acidez encontrada en el presente análisis es superior al valor ideal de 4,2 en ensilajes de buena calidad (Suárez et al., 2011). Estos niveles pueden deberse a que el valor final de pH no está determinado únicamente por la velocidad y cantidad de producción de ácidos, sino también por la presión osmótica (Santos et al., 2010), por lo que al ser los valores de materia seca determinados relativamente más altos que los normales en forrajes tropicales, el material se estabilizó a un nivel menor de acidez.

Todos los tratamientos presentaron características organolépticas deseables en un ensilaje de buena calidad (Maza et al., 2011), además las observaciones encontradas son similares a lo encontrado por Qamar (2009) utilizando melaza y maíz molido como aditivos, así como lo determinado por López et al. (2009) en ensilajes adicionados con melaza y pulpa de cítricos deshidratada. El desempeño del material ensilado en la evaluación organoléptica realizada puede explicarse gracias al aporte de carbohidratos fácilmente fermentables hecho por los aditivos utilizados, lo cual aumenta la posibilidad de conservación del forraje estudiado (Qamar, 2009; Maza et al., 2011).

Finalmente, la composición nutricional del pasto Ratana (Ischaemum indicum Houtt.) fue menor que la de otros forrajes tropicales disponibles, aunque su alto contenido de materia seca y reducida resistencia a la acidificación a causa de su bajo porcentaje de proteína cruda, lo convierte en una muy buena opción para la elaboración de ensilajes que permitan suplementar el faltante de alimento durante la época de escasez; principalmente si el material inicial es mezclado con aditivos como los evaluados, que aumenten la cantidad de carbohidratos no fibrosos disponibles para la fermentación.

\section{AGRADECIMIENTO}

A la Cooperativa de Productores de Leche Dos Pinos R.L. 


\section{LITERATURA CITADA}

AOAC (Association of Analytical Communities) 2000. Official methods of analysis. $17^{\text {th }} \mathrm{ed}$. Washington DC., Estados Unidos.

Araujo-Febres, O., A. Márquez-Araque, O. Ferrer, y A. Pirela. 1996. Evaluación cualitativa de silaje de pasto elefante enano (Pennisetum purpureum cv. Mott) a diferentes edades de corte y adicionando urea y melaza. Rev Fac Agron (LUZ): 13:371-380.

Arosemena, E., D.A. Pezo, D.L. Kass, y P.J. Argel. 1996. Requerimientos externos e internos de fósforo en pasto ratana (Ischaemum indicum (Houtt.) Merrill) y Brachiaria brizantha (A. Rich.) Stapf. Revista Pasturas Tropicales 18(1):34-40.

Bergamaschine, A.F., M. Passipiéri, W.V. Veriano, O.J. Isepon, y L.D. Almeida-Correa. 2006. Qualidade e valor nutritivo de silagens de capim-marandu ( $B$. brizantha cv. Marandu) produzidas com aditivos ou forragem emurchecida. R Bras Zootec 35(4):14541462.

Betancourt, M., I. González, y M. Martínez. 2005. Evaluación de la calidad de los ensilajes. Revista Digital CENIAP HOY 8:1-5.

Bolsen, K.K., G. Ashbell, y Z.G. Wienberg. 1996. Silage fermentation and silage additives. Asian-Australas. J. Anim. Sci. 9(5):483-493.

Botega, V., J. Cardoso, A.R. Evangelista, H. César, P. Figueiredo, C.L. Da-Silva, y R.F. De-Lima. 2009. Efeitos da compactação, da inclusão de aditivo absorvente e do emurchecimento na composição bromatológica de silagens de Capim-Tanzânia. R. Bras. Zootec. 38:40-49.

Ferrari-Junior, E., V.T. Paulino, R.A. Possenti, y T.L. Lucenas. 2009. Aditivos em silagem de capim Elefante Paraíso (Pennisetum hybridum cv. Paraíso). Arch. Zootecn. 58:185-194.

Garcés, A.M., L. Berrio, S. Ruíz, J.G. Serna, y A.F. Builes. 2004. Ensilaje como fuente de alimentación para el ganado. Rev. Lasallista Investig. 1 (001):66-71.

Goering, H.K., y P.J. Van-Soest. 1970. Forage fiber analysis (apparatus, reagents, procedures and some applications). Washington DC., Estados Unidos.

Huisden, C.M., A.T. Adesogan, S.C. Kim, y T. Ososanya. 2009. Effect of applying molasses or inoculants containing homofermentative or heterofermentative bacteria at two rates on the fermentation and aerobic stability of corn silage. J. Dairy Sci. 92:690-697.
Lara, J. 2011. Aditivos para el mejoramiento del ensilaje forrajero. Trabajo presentado para obtener el título de Médico Veterinario Zootecnista. Universidad Michoacana de San Nicolás de Hidalgo, Michoacán, México.

López, M., R. Wingching-Jones, y A. Rojas-Bourrillón. 2009. Características fermentativas y nutricionales del ensilaje de rastrojo de piña (Ananas comosus). Agron. Costarricense 33(1):1-15.

Maza, L., O. Vergara, y E. Paternina. 2011. Evaluación química y organoléptica del ensilaje de Maralfalfa (Pennisetum sp.) más yuca fresca (Manihot esculenta). Rev MVZ Córdoba 16:2528-2537.

Mazza, P.E., L.M. Oliveira, R. Weldert, R. Passini, y P. Marques. 2005. Efeito da adição de níveis crescentes de polpa cítrica sobre a qualidade fermentativa e o valor nutritivo da silagem de Capim-Elefante. R. Bras. Zootec. 34:1138-1145.

Mazza, P.H., J.R. Lobo, E.J. Alves, L.F. Onofre, P. Marques, y J.J. Assumpção. 2007. Efeito da inclusão de polpa cítrica peletizada na confecção de silagem de CapimElefante (Pennisetum purpureum, Schum.). R. Bras. Zootec. 36:1751-1760.

NRC (National Research Council.) 2001. Nutrient requirements of dairy cattle. 7 rev. ed. Washington DC., USA.

$\mathrm{Ng}$, T.T. 1972. Comparative responses of some tropical grasses to fertiliser nitrogen in Sarawak and Malaysia. Trop. Grassl. 6(3):229-236.

Nisa, M., M.A. Shahzad, M. Sarwar, y N.A. Tauqir. 2008. Influence of additives and fermentation periods on silage characteristics, chemical composition, and in situ digestion kinetics of Jambo silage and its fodder in Nili buffalo bulls. Turk. J. Vet. Anim. Sci. 32(2):67-72.

Ojeda, F., I. Montejo, y O. López. 2006. Estudio de la calidad fermentativa de la morera y la hierba de guinea ensiladas en diferentes proporciones. Revista Pastos y Forrajes 29:1-9.

Pereira, R. C., V.L. Banys, A.C. Silva, y R.G.A. Pereira. 1999. Adição de polpa cítrica peletizada na ensilagem de capimelefante (Pennisetum purpureum Schum) cv. Cameroon. Rev. Univ. Alfenas 5:147-152.

Qamar, M. 2009. Effect of molasses and corn as silage additives on the characteristics of Mott Dwarf Elephant grass silage at different fermentation periods. Pak. Vet. J. 29:19-23.

Reyes, N., B. Mendieta, T. Fariñas, M. Mena, J. Cardona, y D. Pezo. 2009. Elaboración y utilización de ensilajes 
en la alimentación del ganado bovino. CATIE, Managua, Nicaragua.

SAS Institute. 1985. SAS user's guide: Statistics. Version 5 ed. SAS Inst., Cary, NC, USA.

Sánchez, J.M., L. Piedra, y H. Soto. 1998. Calidad nutricional de los forrajes en zonas con niveles bajos de produccion de leche, en la zona norte de Costa Rica. Agron. Costarricense 22(1):69-76.

Sánchez, J.M., y H. Soto. 1999. Calidad nutricional de los forrajes de una zona con niveles medios de producción de leche, en el trópico húmedo del norte de Costa Rica. Agron. Costarricense 23(2):165-171.

Santos, M.V.F., A.G. Gómez, J.M. Perea, A. García, A. Guim, y M. Pérez. 2010. Fatores que afetam o valor nutritivo da silagens de forrageiras tropicais. Arch. Zootecn. 59:25-43.

Steel, R., y J. Torrie. 1988. Bioestadística: principios y procedimientos. México DF, México.

Suárez, R., J. Mejía, M. González, D.E. García, y D.A. Perdomo. 2011. Evaluación de ensilajes mixtos de
Saccharum officinarum y Gliricidia sepium con la utilización de aditivos. Revista Pastos y Forrajes 34:69-86.

Van-Soest, P.J., J.B. Robetson, y B.A. Lewis. 1991. Methods for dietary fiber, neutral detergent fiber and non starch polysaccharides in relation to animal nutrition. J. Dairy Sci. 74:3583-3597.

Villarreal, M. 1992. Evaluación comparativa de Ratana (lschaemum ciliare) como especie forrajera. Agron. Costarricense 16(1):37-44.

Villareal, M. 2010. Respuesta del pasto Ratana (Ischaemun indicum) a la fertilización nitrogenada. http://corfoga. org/2012/wp-content/uploads/2012/10/Respuestadel-Pasto-Retana-a-la-Fertilizacion-NitrogenadaMAG-ITCR-CORFOGA.pdf. (Consultado 24 enero 2013).

Wattiaux, M. s.f. Introducción al proceso de ensilaje. http://babcock.wisc.edu/sites/default/files/documents/ productdownload/du_502.es_.pdf (Consultado 23 enero 2013). 
